Supplement of Geosci. Model Dev., 11, 2581-2608, 2018

https://doi.org/10.5194/gmd-11-2581-2018-supplement

(C) Author(s) 2018. This work is distributed under

the Creative Commons Attribution 4.0 License.

(c) (1)

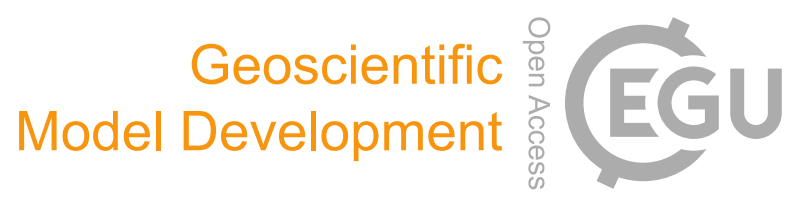

Supplement of

\title{
The Interactive Stratospheric Aerosol Model Intercomparison Project (ISA-MIP): motivation and experimental design
}

Claudia Timmreck et al.

Correspondence to: Claudia Timmreck (claudia.timmreck@mpimet.mpg.de)

The copyright of individual parts of the supplement might differ from the CC BY 4.0 License. 


\begin{tabular}{|l|l|}
\hline Specifications & Reference \\
\hline Greenhouse gases ODPs & As recommended for the SPARC CCMI hindcast scenario \\
& REF-C1SD (Eyring et al, 2013) \\
http://www.met.reading.ac.uk/ccmi/?page_id=11
\end{tabular}

Table S1: Overview of background conditions.

\begin{tabular}{|l|l|}
\hline Sulphur emission & Reference \\
\hline $\mathrm{SO}_{2}$ Anthropogenic & $\begin{array}{l}\text { From MACC-CITY (Granier et al., 2011) for time period considered and as } \\
\text { extended back to 1960 on ECCAD website } \\
\text { http://eccad.sedoo.fr/eccad_extract_interface/JSF/page_login.jsf }\end{array}$ \\
\hline $\mathrm{SO}_{2}$ Biomass burning & $\begin{array}{l}\text { Biomass burning: GFEDv4 (http://www.globalfiredata.org/index.html) } \\
\text { From MACC-CITY (Granier et al., 2011) for time period considered and as } \\
\text { extended back to 1960 on ECCAD website) }\end{array}$ \\
\hline $\begin{array}{l}\text { Continuously degassing } \\
\text { volcanoes }\end{array}$ & $\begin{array}{l}\text { "continuous_volc.1x1" from Aerocom-I (Dentener et al., 2006) based on Andres } \\
\text { and Kasgnoc (1998) which presents an average estimate of the contribution of } \\
\text { silent degassing volcanoes to the global sulphur budget, } \\
\text { http://aerocom.met.no/download/emissions/AEROCOM_B-PRE/other_ascii// }\end{array}$ \\
\hline DMS & $\begin{array}{l}\text { Sea water concentration from Lana et al. (2011) is recommended } \\
\text { https://www.bodc.ac.uk/solas_integration/implementation_products/group1/dms/ } \\
\text { Biogenic modeller's choice }\end{array}$ \\
\hline OCS & $\begin{array}{l}\text { Concentrations are fixed at surface and equal to 510 pptv (Montzka et al., 2013; } \\
\text { ASAP2006) }\end{array}$ \\
\hline
\end{tabular}

Table S2: Overview of sulphur emission.

\begin{tabular}{|l|l|}
\hline Name & Description \\
\hline nh_50 & $\begin{array}{l}\text { Passive tracer with fix surface concentration equal to } 100 \text { ppb between } 30^{\circ} \mathrm{N} \text { and } \\
50^{\circ} \mathrm{N} \text { and equal to 0 outside of this latitudinal band, e-folding decay time of } 50 \text { days }\end{array}$ \\
\hline tr_50 & $\begin{array}{l}\text { Passive tracer with fix surface concentration equal to } 100 \mathrm{ppb} \text { between } 20^{\circ} \mathrm{S} \text { and } \\
20^{\circ} \mathrm{N} \text { and equal to } 0 \text { outside of this latitudinal band, e-folding decay time of } 50 \text { days; }\end{array}$ \\
\hline sh_50 & $\begin{array}{l}\text { Passive tracer with fix surface concentration equal to } 100 \mathrm{ppb} \text { between } 50^{\circ} \mathrm{S} \text { and } 30^{\circ} \mathrm{S} \\
\text { and equal to } 0 \text { outside of this latitudinal band, e-folding decay time of } 50 \text { days. }\end{array}$ \\
\hline AOA & $\begin{array}{l}\text { Passive tracer for the stratospheric mean age-of-air. Modelling groups can use their } \\
\text { existing implementation or implement a tracer with a global fixed surface layer } \\
\text { mixing ratio of 0 ppbv and a uniform unspecified fixed source (at all levels) } \\
\text { everywhere else, which must be constant in space and time. }\end{array}$ \\
\hline ST80_25 & $\begin{array}{l}\text { Passive tracer to estimate the exchange from the stratosphere to the troposphere. This } \\
\text { is achieved by fixing the mixing ratio above 80hPa (200ppbv) to a constant value, } \\
\text { and imposing a uniform fixed 25-day exponential decay in the troposphere only. }\end{array}$ \\
\hline Volc & $\begin{array}{l}\text { Passive volcanic tracer for the HerSEA experiments. The tracer is initialized in the } \\
\text { same way as the volcanic } \mathrm{SO}_{2} \text { emission, with an initial value of } 1 .\end{array}$ \\
\hline
\end{tabular}

Table S3: Suggested passive tracers mostly following the CCM protocol (Eyring et al., 2013). 


\begin{tabular}{|c|c|c|c|c|}
\hline Long name & $\begin{array}{c}\text { Variable } \\
\text { name }\end{array}$ & Unit & Category & Comment \\
\hline grid-cell area & area & $\mathrm{m}^{2}$ & 1 & \\
\hline land fraction & landf & 1 & 1 & $\begin{array}{l}\text { Please express "X_area_fraction" as the } \\
\text { fraction of horizontal area occupied by X. }\end{array}$ \\
\hline surface altitude & orog & $\mathrm{m}$ & 1 & $\begin{array}{l}\text { "Surface" means the lower boundary of } \\
\text { the atmosphere. Altitude is the (geometric) } \\
\text { height above the geoid, which is the } \\
\text { reference geopotential surface. }\end{array}$ \\
\hline \multicolumn{5}{|c|}{ Meteorology } \\
\hline Precipitation & precip & $\mathrm{kg} \mathrm{m}^{-2} \mathrm{~s}^{-1}$ & 1 & $\begin{array}{c}\text { Includes all types: rain, snow, large-scale, } \\
\text { convective, etc. }\end{array}$ \\
\hline surface temperature & tas & $\mathrm{K}$ & 1 & \\
\hline surface air pressure & ps & $\mathrm{Pa}$ & 1 & $\begin{array}{l}\text { "Surface" means the lower boundary of } \\
\text { the atmosphere. }\end{array}$ \\
\hline Cloud fraction & clt & $\%$ & 1 & Cloud fraction as seen from top or surface \\
\hline tropopause_air_pressure & ptp & $\mathrm{Pa}$ & 2 & $\begin{array}{c}\text { 2D monthly mean thermal tropopause } \\
\text { calculated using WMO tropopause } \\
\text { definition on } 3 \mathrm{~d} \text { temperature }\end{array}$ \\
\hline tropopause_air_temperature & tatp & $\mathrm{K}$ & 2 & See above \\
\hline tropopause_altitude & ztp & $\mathrm{M}$ & 2 & See above \\
\hline \multicolumn{5}{|c|}{ Budget } \\
\hline Load of H2SO4 (aerosol) & loadso4 & $\mathrm{kg} \mathrm{m}^{-2}$ & 1 & $\begin{array}{c}\text { Units of the particle-phase-sulphur should } \\
\text { be using mass of } \mathrm{H} 2 \mathrm{SO} 4\end{array}$ \\
\hline Load of SO2(g) & loadso2 & $\mathrm{kg} \mathrm{m}^{-2}$ & 1 & \\
\hline Load of $\mathrm{H} 2 \mathrm{SO} 4(\mathrm{~g})$ & loadh2so4 & $\mathrm{kg} \mathrm{m}^{-2}$ & 1 & \\
\hline Load of OCS & loadocs & $\mathrm{kg} \mathrm{m}^{-2}$ & 1 & \\
\hline Load of DMS & loaddms & $\mathrm{kg} \mathrm{m}^{-2}$ & 2 & \\
\hline Load of H2S & loadh2s & $\mathrm{kg} \mathrm{m}^{-2}$ & 3 & \\
\hline Load of CS2 & loades2 & $\mathrm{kg} \mathrm{m}^{-2}$ & 3 & \\
\hline \multicolumn{5}{|c|}{ Removal } \\
\hline dry deposition of DMS & drysdms & $\mathrm{kg} \mathrm{m}^{-2} \mathrm{~s}^{-1}$ & 2 & \\
\hline dry deposition of $\mathrm{SO} 2$ & dryso2 & $\mathrm{kg} \mathrm{m}^{-2} \mathrm{~s}^{-1}$ & 1 & \\
\hline dry deposition of $\mathrm{H} 2 \mathrm{SO} 4(\mathrm{~g})$ & dryh2so4 & $\mathrm{kg} \mathrm{m}^{-2} \mathrm{~s}^{-1}$ & 1 & \\
\hline dry deposition of $\mathrm{H} 2 \mathrm{SO} 4(\mathrm{p})$ & dryso4 & $\mathrm{kg} \mathrm{m}^{-2} \mathrm{~s}^{-1}$ & 1 & \\
\hline sedimentation of SO4 & sedso4 & $\mathrm{kg} \mathrm{m}^{-2} \mathrm{~s}^{-1}$ & 1 & \\
\hline dry deposition of $\mathrm{H} 2 \mathrm{~S}$ & dryh2s & $\mathrm{kg} \mathrm{m}^{-2} \mathrm{~s}^{-1}$ & 2 & \\
\hline dry deposition of $\mathrm{C} 2 \mathrm{~S}$ & dryc2s & $\mathrm{kg} \mathrm{m}^{-2} \mathrm{~s}^{-1}$ & 2 & \\
\hline wet deposition of SO2 & wetso2 & $\mathrm{kg} \mathrm{m}^{-2} \mathrm{~s}^{-1}$ & 1 & \\
\hline wet deposition of H2SO4(p) & wetso4 & $\mathrm{kg} \mathrm{m}^{-2} \mathrm{~s}^{-1}$ & 1 & \\
\hline wet deposition of DMS & wetdms & $\mathrm{kg} \mathrm{m}-{ }^{2} \mathrm{~s}^{-1}$ & 2 & \\
\hline wet deposition of $\mathrm{C} 2 \mathrm{~S}$ & wetc2s & $\mathrm{kg} \mathrm{m}^{-2} \mathrm{~s}^{-1}$ & 2 & \\
\hline wet deposition of $\mathrm{H} 2 \mathrm{~S}$ & weth2s & $\mathrm{kg} \mathrm{m}^{-2} \mathrm{~s}^{-1}$ & 2 & \\
\hline \multicolumn{5}{|c|}{ Emission } \\
\hline total emission of $\mathrm{SO} 2$ & emiso2 & $\mathrm{kg} \mathrm{m}^{-2} \mathrm{~s}^{-1}$ & 1 & \\
\hline total emission of DMS & emidms & $\mathrm{kg} \mathrm{m}^{-2} \mathrm{~s}^{-1}$ & 2 & \\
\hline total emission of COS & emicos & $\mathrm{kg} \mathrm{m}-2 \mathrm{~s}-1$ & 1 & If available \\
\hline total emission of DMS & emih2s & $\mathrm{kg} \mathrm{m}-2 \mathrm{~s}-1$ & 1 & \\
\hline total emission of CS2 & emic2s & $\mathrm{kg} \mathrm{m}-2 \mathrm{~s}-1$ & 3 & \\
\hline \multicolumn{5}{|c|}{ Fluxes } \\
\hline So2 Flux to the tropopause & flxso2 & $\mathrm{kg} \mathrm{m}^{-2} \mathrm{~s}^{-1}$ & 1 & \\
\hline H2SO4(p)Flux through the tropoause (total) & flxso4t & $\mathrm{kg} \mathrm{m}^{-2} \mathrm{~s}^{-1}$ & 1 & \\
\hline $\begin{array}{c}\text { H2SO4 Flux (tropopause) per size } \\
\text { class/modes }\end{array}$ & flxso4_ & $\mathrm{kg} \mathrm{m}^{-2} \mathrm{~s}^{-1}$ & 3 & \\
\hline Flux H2SO4 $(\mathrm{p})>5 \mathrm{~nm}$ & flxso4p150 & $\mathrm{kg} \mathrm{m}^{-2} \mathrm{~s}^{-1}$ & 2 & \\
\hline Flux H2SO4 (p) $>150 \mathrm{~nm}$ & flxso4p150 & $\mathrm{kg} \mathrm{m}^{-2} \mathrm{~s}^{-1}$ & 2 & \\
\hline Flux H2SO4 (p) >250nm & flxso4p250 & $\mathrm{kg} \mathrm{m}^{-2} \mathrm{~s}^{-1}$ & 2 & \\
\hline Flux H2SO4 (p) $>550 \mathrm{~nm}$ & flxso4p550 & $\mathrm{kg} \mathrm{m}^{-2} \mathrm{~s}^{-1}$ & 2 & \\
\hline Flux H2SO4 (p) >750nm & flxso4p750 & $\mathrm{kg} \mathrm{m}^{-2} \mathrm{~s}^{-1}$ & 2 & \\
\hline Flux H2SO4 (p) >1000nm & flxso4p1000 & $\mathrm{kg} \mathrm{m}^{-2} \mathrm{~s}^{-1}$ & 2 & \\
\hline
\end{tabular}




\begin{tabular}{|c|c|c|c|c|}
\hline \multicolumn{5}{|c|}{ Radiation } \\
\hline AOD @ 386nm & od386aer & 1 & 2 & \\
\hline AOD @ 453nm & od453aer & 1 & 2 & \\
\hline AOD @ 525nm & od525aer & 1 & 1 & \\
\hline AOD @ 750nm & od750aer & 1 & 2 & \\
\hline AOD @ 870nm & pd870aer & 1 & 2 & \\
\hline AOD @ 1020nm & od1020aer & 1 & 1 & \\
\hline AOD @3460nm & od3460aer & 1 & 2 & \\
\hline AOD @5260nm & od5260aer & 1 & 2 & \\
\hline AOD@ 12660nm & od5260aer & 1 & 2 & \\
\hline Surface downwelling SW radiation & rsds & $\mathrm{W} \mathrm{m}^{-2}$ & 1 & \\
\hline Surface upwelling SW radiation & rsus & $\mathrm{W} \mathrm{m}^{-2}$ & 1 & \\
\hline Surface downwelling LW radiation & rlds & $\mathrm{W} \mathrm{m}^{-2}$ & 1 & \\
\hline Surface upwelling LW radiation & rldus & $\mathrm{W} \mathrm{m}^{-2}$ & 1 & \\
\hline Surface downwelling SW flux clear sky & rsdscs & $\mathrm{W} \mathrm{m}^{-2}$ & 2 & \\
\hline Surface upwelling SW flux clear sky & rsuscs & $\mathrm{W} \mathrm{m}^{-2}$ & 2 & \\
\hline Surface upwelling LW flux clear sky & rldcs & $\mathrm{W} \mathrm{m}^{-2}$ & 2 & \\
\hline Surface diffuse SW flux & rsdsdiff & $\mathrm{W} \mathrm{m}^{-2}$ & 2 & \\
\hline Surface diffuse SW flux clear sky & rsdscsdiff & $\mathrm{W} \mathrm{m}^{-2}$ & 2 & \\
\hline TOA Incident & rst & $\mathrm{W} \mathrm{m}^{-2}$ & 2 & \\
\hline TOA downwelling SW radiation & rsdt & $\mathrm{W} \mathrm{m}^{-2}$ & 1 & \\
\hline TOA downwelling LW radiation & rldt & $\mathrm{W} \mathrm{m}^{-2}$ & 1 & \\
\hline TOA outgoing SW radiation & rsut & $\mathrm{W} \mathrm{m}^{-2}$ & 1 & \\
\hline TOA outgoing SW radiation clear sky & rsutcs & $\mathrm{W} \mathrm{m}^{-2}$ & 2 & \\
\hline TOA outgoing LW radiation & rlut & $\mathrm{W} \mathrm{m}^{-2}$ & 1 & \\
\hline TOA outgoing LW radiation clear sky & rlutcs & $\mathrm{W} \mathrm{m}^{-2}$ & 2 & \\
\hline Total photsynthtically FLUX (PAR) & tphotpar & $\mathrm{W} \mathrm{m}^{-2}$ & 3 & \\
\hline photsynthtically FLUX (PAR) & photpar & $\mathrm{W} \mathrm{m}^{-2}$ & 3 & \\
\hline
\end{tabular}

Table S4: Overview of two-dimensional variables requested for ISA-MIP following mainly the AEROCOM protocols: http://aerocom.met.no/protocol.html. (1) indicates mandatory variables, which are in addition shaded, (2) important variables but not required, (3) values which are nice to have for special diagnostic. Monthly mean output is satisfactory except for the meteorological values, which should be provided in daily resolution. 


\begin{tabular}{|c|c|c|c|c|}
\hline Long name & $\begin{array}{l}\text { Variable } \\
\text { name }\end{array}$ & Unit & Category & Comment \\
\hline \multicolumn{5}{|c|}{ Meteorology } \\
\hline air temperature & ta & $\mathrm{K}$ & 1 & $\begin{array}{l}\text { Air temperature is the bulk temperature of the air, } \\
\text { not the surface (skin) temperature. }\end{array}$ \\
\hline specific humidity & hus & 1 & 1 & $\begin{array}{l}\text { Specific means per unit mass. Specific humidity is } \\
\text { the mass fraction of water vapor in (moist) air. }\end{array}$ \\
\hline air mass & airmass & $\mathrm{kg} \mathrm{m}^{-2}$ & 1 & Vertically integrated mass content of air in layer \\
\hline pressure & pfull & $\mathrm{Pa}$ & 1 & Air pressure on model levels \\
\hline zonal wind & ua & $\mathrm{m} / \mathrm{s}$ & 1 & \\
\hline meridional wind & va & $\mathrm{m} / \mathrm{s}$ & 1 & \\
\hline vertical wind & wa & $\mathrm{m} / \mathrm{s}$ & 1 & \\
\hline geopotential height & $\mathrm{Zg}$ & $\mathrm{m}$ & 1 & \\
\hline cloud fraction & clt3D & $\%$ & 2 & \\
\hline cloud optical depth & $\operatorname{cod} 3 \mathrm{D}$ & 1 & 2 & \\
\hline aerosol water & mmraerh2o & 1 & 3 & \\
\hline convective updraft mass flux & mcu & $\mathrm{kg} \mathrm{m}-^{2} \mathrm{~s}^{-1}$ & 3 & $\begin{array}{l}\text { The atmosphere convective mass flux is the } \\
\text { vertical transport of mass for a field of cumulus } \\
\text { clouds or thermals, given by the product of air } \\
\text { density and vertical velocity. For an area-average, } \\
\text { cell_methods should specify whether the average } \\
\text { is over all the area or the area of updrafts only. }\end{array}$ \\
\hline \multicolumn{5}{|c|}{ Sulfur Chemistry } \\
\hline OCS & vmrocs & 1 & 1 & \\
\hline $\mathrm{SO} 2$ & vmrso2 & 1 & 2 & \\
\hline DMS & vmrdms & 1 & 2 & \\
\hline $\mathrm{H} 2 \mathrm{~S}$ & vmr h2s & 1 & 3 & \\
\hline $\mathrm{H} 2 \mathrm{SO} 4(\mathrm{~g})$ & vmrh2so4 & 1 & 2 & \\
\hline $\mathrm{CS} 2$ & vmres & 1 & 3 & \\
\hline $\mathrm{SO} 3$ & vmrso3 & 1 & 2 & \\
\hline H2SO4 (p) total) & mmso4r & 1 & 1 & Mass mixing ratio of sulphate mass (total) \\
\hline \multicolumn{5}{|c|}{ Mass mixing ratio of sulfate mass in each size class } \\
\hline $\mathrm{H} 2 \mathrm{SO} 4(\mathrm{p})>5 \mathrm{~nm}$ & $\mathrm{mmso} 4 \mathrm{r} 5$ & 1 & 2 & $\mathrm{OPC}$ \\
\hline $\mathrm{H} 2 \mathrm{SO} 4(\mathrm{p})>150 \mathrm{~nm}$ & mmso4r15 & 1 & 2 & $\mathrm{OPC}$ \\
\hline $\mathrm{H} 2 \mathrm{SO} 4(\mathrm{p})>250 \mathrm{~nm}$ & mmso4r25 & 1 & 2 & OPC \\
\hline $\mathrm{H} 2 \mathrm{SO} 4(\mathrm{p})>550 \mathrm{~nm}$ & mmso4r55 & 1 & 2 & OPC \\
\hline $\mathrm{H} 2 \mathrm{SO} 4(\mathrm{p})>750 \mathrm{~nm}$ & mmso4r75 & 1 & 2 & OPC \\
\hline $\mathrm{H} 2 \mathrm{SO} 4(\mathrm{p})>1000 \mathrm{~nm}$ & mmso4r100 & 1 & 2 & $\mathrm{OPC}$ \\
\hline \multicolumn{5}{|c|}{ Microphysical processes } \\
\hline number formation through nucleation & nucpn & $\mathrm{m}^{-3} \mathrm{~s}^{-1}$ & 2 & \\
\hline sedimentation of SO4 & sedso4 & $\mathrm{kg} \mathrm{m}^{-2} \mathrm{~s}^{-1}$ & 2 & Net downward (out-below minus in-above) \\
\hline $\mathrm{H} 2 \mathrm{SO} 4$ condensation flux & conh2so4 & $\mathrm{kg} \mathrm{m}^{-2} \mathrm{~s}^{-1}$ & 2 & Net transfer into the particulate phase \\
\hline \multicolumn{5}{|c|}{ Chemistry } \\
\hline $\mathrm{N} 2 \mathrm{O}$ & vmrn2o & 1 & 3 & \\
\hline $\mathrm{OH}$ & vmroh & 1 & 1 & \\
\hline $\mathrm{O} 3$ & vmro3 & 1 & 1 & \\
\hline HNO3 & vmrhno3 & 1 & 3 & \\
\hline NO & vmrno & 1 & 3 & \\
\hline $\mathrm{NO} 2$ & vmrno2 & 1 & 3 & \\
\hline $\mathrm{N} 2 \mathrm{O} 5$ & vmrn2o5 & 1 & 3 & \\
\hline \multicolumn{5}{|c|}{ Bulk parameters } \\
\hline surface area density & sad & $\mathrm{m}^{2} / \mathrm{m}^{3}$ & 1 & \\
\hline effective radius & reff & M & 1 & \\
\hline \multicolumn{5}{|c|}{ Particle numbers } \\
\hline $\mathrm{N}$ total & concen & $\mathrm{m}^{-3}$ & 1 & number_concentration_of_ambient_aerosol_in_air \\
\hline $\mathrm{N}>5 \mathrm{~nm}$ & conc5 & $\mathrm{m}^{-3}$ & 2 & $\mathrm{CPC}$ \\
\hline $\mathrm{N}>150 \mathrm{~nm}$ & conc 150 & $\mathrm{~m}^{-3}$ & 2 & $\mathrm{OPC}$ \\
\hline $\mathrm{N}>250 \mathrm{~nm}$ & conc 250 & $\mathrm{~m}^{-3}$ & 2 & $\mathrm{OPC}$ \\
\hline $\mathrm{N}>550 \mathrm{~nm}$ & conc550 & $\mathrm{m}^{-3}$ & 2 & $\mathrm{OPC}$ \\
\hline $\mathrm{N}>750 \mathrm{~nm}$ & conc750 & $\mathrm{m}^{-3}$ & 2 & $\mathrm{OPC}$ \\
\hline
\end{tabular}




\begin{tabular}{|c|c|c|c|c|}
\hline $\mathrm{N}>1000 \mathrm{~nm}$ & conc1000 & $\mathrm{m}^{-3}$ & 2 & OPC \\
\hline \multicolumn{5}{|c|}{ Extinction } \\
\hline Aerosol extinction @386nm & ec386aer & $\mathrm{m}^{-1}$ & 2 & SAGEII/III, (POAM, shipborne lidar) \\
\hline Aerosol extinction @440nm & ec440aer & $\mathrm{m}^{-1}$ & 3 & \\
\hline Aerosol extinction@525nm & ec525aer & $\mathrm{m}^{-1}$ & 1 & SAGE-II \\
\hline Aerosol extinction@750nm & ec750aer & $\mathrm{m}^{-1}$ & 2 & OSIRIS \\
\hline Aerosol extinction@870nm & ec870aer & $\mathrm{m}^{-1}$ & 3 & \\
\hline Aerosol extinction @1020nm & ec1020aer & $\mathrm{m}^{-1}$ & 1 & SAGEII \\
\hline Aerosol extinction @3460nm & ec3460aer & $\mathrm{m}^{-1}$ & 2 & HALOE \\
\hline Aerosol extinction @5260nm & ec5260aer & $\mathrm{m}-1$ & 2 & HALOE \\
\hline aerosol extinction@12660nm & ec12660aer & $\mathrm{m}^{-1}$ & 3 & ISAMS \\
\hline \multicolumn{5}{|c|}{ Absorption } \\
\hline aerosol absorption@386nm & abs386aer & $\mathrm{m}^{-1}$ & 3 & SAGEII/III, (POAM, shipborne lidar) \\
\hline aerosol absorption@440nm & abs440aer & $\mathrm{m}^{-1}$ & 3 & \\
\hline aerosol absorption @525nm & abs525aer & $\mathrm{m}^{-1}$ & 2 & SAGE-II \\
\hline aerosol absorption@750nm & abs750aer & $\mathrm{m}^{-1}$ & 3 & OSIRIS \\
\hline aerosol absorption @870nm & abs870aer & $\mathrm{m}^{-1}$ & 3 & \\
\hline aerosol absorption @1020nm & abs1020aer & $\mathrm{m}^{-1}$ & 2 & SAGE-II \\
\hline aerosol absorption@3460nm & abs3460aer & $\mathrm{m}^{-1}$ & 3 & HALOE \\
\hline aerosol absorption @ 5260nm & abs5260aer & $\mathrm{m}^{-1}$ & 3 & HALOE \\
\hline aerosol absorption@12660nm & abs12660aer & $\mathrm{m}^{-1}$ & 3 & ISAMS \\
\hline asymmetry factor@525nm & asy525aer & 1 & 1 & \\
\hline
\end{tabular}

Table S5: Overview of three-dimensional variables requested for ISA-MIP following mainly the AEROCOM protocols: http://aerocom.met.no/protocol.html. All 3D data to be provided on either host model vertical levels or preferably (if resources allow) on the reference pressure levels 1000, 925, 850, 700, 600, 500, 400, 300, 250,200, 150, $100,70,50,30,20 \& 10 \mathrm{hPa}$. If possible also on the additional pressure levels: 7, 5, 3, 2, 1 and $0.4 \mathrm{hPa}$. (1) indicates mandatory variables, which are in addition shaded, (2) important variables but not required, (3) values which are nice to have for special diagnostic. Monthly mean output is satisfactory except for the meteorological values, which should be provided in daily resolution.

\begin{tabular}{|c|c|c|c|c|c|c|}
\hline Volcano & Lon & Lat & Time & $\begin{array}{c}\text { Min Plume } \\
\text { Height }(\mathbf{k m})\end{array}$ & $\begin{array}{c}\text { Max Plume } \\
\text { Height (km) }\end{array}$ & $\begin{array}{c}\text { Mean SO2 } \\
(\mathbf{k t})\end{array}$ \\
\hline Manam & 145.04 & -4.08 & 27 Jan 2005 & 18 & 24 & 154.67 \\
\hline Soufriere Hills & 297.82 & $16.72 ;$ & 19 May 2006;; & 19 & 20 & 185.33 \\
\hline Rabaul/Tavurvur & 152.20 & $-4.27 ;$ & 7 Oct 2006 & 17 & 18 & 234.0 \\
\hline Okmok & 168.10 & 53.43 & 12 Jul 2008 & 10 & 16 & 109.0 \\
\hline Kasatochi & 175.50 & 52.18 & 7 Aug 2008 & 10 & 18 & 1363.33 \\
\hline Sarychev & 153.20 & 48.09 & 15 Jun 2009 & 11 & 17 & 965.33 \\
\hline Merapi & 110.44 & -7.54 & 4 Nov 2010 & 14 & 17 & 282.67 \\
\hline Nabro & 41.70 & $13.37 ;$ & 13 Jun 2011 & 9.7 & 18 & 1307.0 \\
\hline
\end{tabular}

Table S6: Overview of VolcDSUB, a subset of volcanic emissions, that were derived based on the average mass of $\mathrm{SO}_{2}$ emitted using VolcDB1, VolcDB2, and VolcDB3. (http://isamip.eu/fileadmin/user_upload/isamip/volc_sub_v185.dat). 


\section{References}

Andres, R. J. and Kasgnoc, A. D.: A time-averaged inventory of subaerial volcanic sulfur emissions, J. Geophys. Res., 103, 25251-25261, 1998.

Bingen, C., Robert, C. E., Stebel, K., Brühl, C., Schallock, J., Vanhellemont, F., Mateshvili N., Höpfner, M., Trickl, T., Barnes, J.E., Jumelet, J., Vernier, J.-P. ,Popp T, Gerrit de Leeuw, G., Pinnock, S.: Stratospheric aerosol data records for the climate change initiative: Development, validation and application to chemistryclimate modelling. Remote Sensing of Environment. https://doi.org/10.1016/j.rse.2017.06.002, 2017

Brühl, C., Lelieveld, J., Tost, H., Höpfner, M, and Glatthor, N.: Stratospheric sulphur and its implications for radiative forcing simulated by the chemistry climate model EMAC, J. Geophys. Res.-Atmos., 120, 2103-2118, doi:10.1002/2014JD022430, 2015.

Dentener, F., Kinne, S., Bond, T., Boucher, O., Cofala, J., Generoso, S., Ginoux, P., Gong, S., Hoelzemann, J. J., Ito, A., Marelli, L., Penner, J. E., Putaud, J.-P., Textor, C., Schulz, M., van der Werf, G. R., and Wilson, J.: Emissions of primary aerosol and precursor gases in the years 2000 and 1750 prescribed data-sets for AeroCom, Atmos. Chem. Phys., 6, 4321-4344, doi:10.5194/acp-6-4321-2006, 2006.

Eyring, V., J.-F. Lamarque et al., Overview of IGAC/SPARC Chemistry-Climate Model Initiative (CCMI) Community Simulations in Support of Upcoming Ozone and Climate Assessments, SPARC Newsletter No. 40, p. 48-66, 2013

Grams, G. and Fiocco, G.: Stratospheric Aerosol Layer during 1964 and 1965, J. Geophys. Res., vol. 72(14), 3523-3542, 1967.

Granier, C., Bessagnet, B., Bond, T. C., D’Angiola, A., Denier van der Gon, H., Frost, G. J., Heil, A., Kaiser, J. W., Kinne, S., Klimont, Z., Kloster, S., Lamarque, J.-F., Liousse, C., Masui, T., Meleux, F., Mieville, A., Ohara, T., Raut, J.-C., Riahi, K., Schultz, M. G., Smith, S. J., Thompson, A., Aardenne, J.,Werf, G. R., and Vuuren, D. P.: Evolution of anthropogenic and biomass burning emissions of air pollutants at global and regional scales during the 1980-2010 period. Climatic Change, 109, 163-190, DOI: 10.1007/s10584-011-0154-1, 2011.

Lana, A., Bell, T. G., Sinó, R., Vallina, S. M., Ballabrera-Poy, J., Kettle, A. J., Dachs, J., Bopp, L., Saltzman, E. S., Stefels, J., Johnson, J. E., and Liss, P. S.: An updated climatologyof surface dimethlysulfide concentrations and emission fluxesin the global ocean, Global Biogeochem. Cy., 25, GB1004,doi:10.1029/2010gb003850, 2011.

Montzka, S. A., Calvert, P., Hall, B. D., Elkins, J. W., Conway, T. J., Tans, P. P., and Sweeney, C.: On the global distribution, seasonality, and budget of atmospheric carbonyl sulfide and some similarities with $\mathrm{CO} 2$, J. Geophys. Res., 112, D09302, doi:10.1029/2006JD007665, 2007.

Rayner, N. A., D. E. Parker, E. B. Horton, C. K. Folland, L. V. Alexander, D. P. Rowell, E. C. Kent, and A. Kaplan, Global analyses of sea surface temperature, sea ice, and night marine air temperature since the late nineteenth century, J. Geophys. Res.,108 14), 4407, doi:10.1029/2002JD002670, 2003.

SPARC: Assessment of Stratospheric Aerosol Properties (ASAP), SPARC Report No. 4, edited by: Thomason, L. and Peter, T., World Climate Research Programme WCRP-124, WMO/TD No. 1295, 2006. 